\section{Regards sur l'économie allemande}

Bulletin économique du CIRAC

$81 \mid 2007$

Varia

\title{
De la difficulté d'aborder le marché allemand : le cas de Levi Strauss Signature
}

Ulrike Mayrhofer et Claire Roederer

\section{(2) OpenEdition}

\section{Journals}

Édition électronique

URL : http://journals.openedition.org/rea/666

DOI : $10.4000 /$ rea.666

ISBN : 978-2-8218-0858-4

ISSN : 1965-0787

\section{Éditeur}

CIRAC

\section{Édition imprimée}

Date de publication : 1 mai 2007

Pagination : 27-34

ISSN : 1156-8992

Référence électronique

Ulrike Mayrhofer et Claire Roederer, « De la difficulté d'aborder le marché allemand : le cas de Levi Strauss Signature », Regards sur l'économie allemande [En ligne], 81 | mai 2007, document 4, mis en ligne le 01 mai 2009, consulté le 01 mai 2019. URL : http://journals.openedition.org/rea/666 ; DOI : $10.4000 /$ rea.666 


\section{De la difficulté d'aborder le marché allemand : le cas de Levi Strauss Signature}

\author{
Ulrike Mayrhofer et Claire Roederer
}

Dans un contexte de mondialisation économique, les entreprises sont contraintes de s'adapter aux mutations du marché et d'anticiper de nouvelles tendances. Le secteur du textile-habillement est plus particulièrement touché par le phénomène : de nouveaux concurrents apparaissent, et les comportements de consommation évoluent rapidement. C'est dans ce contexte que le groupe américain décide de lancer la nouvelle marque Levi Strauss Signature ${ }^{\circledR}$. L'objectif principal consiste à vendre des jeans en grande distribution : "sell jeans where people shop ". Après l'introduction de la marque en Amérique du Nord, en Australie et au Japon, le groupe décide de conquérir le marché européen. Avec des résultats variables suivant les pays : l'Allemagne semble un marché particulièrement difficile à appréhender pour le groupe américain ...

\section{Levi Strauss, un groupe américain à forte image dans les vêtements de marque}

Fondé en 1853, le groupe Levi Strauss peut être considéré comme une entreprise leader sur le marché des vêtements de marque. En 1873, son fondateur, Levi Strauss, et le tailleur Jacob Davis ont déposé un brevet aux Etats-Unis pour fabriquer des vêtements en denim, créant ainsi le premier "blue jean ». Aujourd'hui, l'entreprise conçoit et commercialise des jeans, des pantalons, des T-Shirts, des vestes et des accessoires pour hommes, femmes et enfants. Elle possède trois marques : Levi's ${ }^{\circledR}\left(71 \%\right.$ du chiffre d'affaires), Dockers ${ }^{\circledR}(19 \%)$ et Levi Strauss Signature ${ }^{\circledR}(10 \%)$.

\begin{tabular}{|c|c|}
\hline \multicolumn{2}{|r|}{ Le groupe Levi Strauss, créateur du jean } \\
\hline 1873 & Levi Strauss \& Co. crée les premiers blue jeans dans le monde. \\
\hline 1890 & Le numéro « 501 » est attribué aux jeans Levi's ${ }^{\circledR}$. \\
\hline 1935 & Introduction de « Lady Levi's ${ }^{\circledR} »$ les premiers jeans de la marque pour femmes. \\
\hline 1986 & $\begin{array}{l}\text { Lancement de Dockers }{ }^{\circledR} \text {, une marque qui a connu une forte croissance et qui se situe } \\
\text { entre le jeans et le costume de ville. La marque change les habitudes vestimentaires des } \\
\text { employés aux Etats-Unis. }\end{array}$ \\
\hline 2000 & $\begin{array}{l}\text { Le magazine Time nomme les jeans Levi's }{ }^{\circledR} 501 \text { comme la meilleure mode du } X X^{e} \text { siècle, } \\
\text { devant la mini-jupe et la petite robe noire. }\end{array}$ \\
\hline 2003 & $150^{\mathrm{e}}$ anniversaire de l'entreprise ; création de la marque Levi Strauss Signature ${ }^{\circledR}$. \\
\hline
\end{tabular}

Le siège social du groupe se trouve à San Francisco. Le capital est principalement détenu par les descendants de la famille de Levi Strauss. Le groupe emploie 9635 salariés, dont 6380 hors des Etats-Unis (35 pays). En 2006, son chiffre d'affaires s'élève à 4,1 milliards $\$$, et le résultat net est de 156 millions $\$$. Les produits du groupe sont commercialisés auprès de 55000 magasins dans 110 pays. Les activités sont organisées selon trois régions géographiques: Amérique du Nord (59\% du chiffre d'affaires), Europe (24\%) et Asie-Pacifique (intégrant également l'Amérique Latine, le Moyen Orient et l'Afrique : $17 \%$ ).
Une entreprise leader sur le marché des vêtements de marque ...

... et qui commercialise ses produits dans 110 pays 
Levi's $^{\circledast}$ : la principale marque de l'entreprise

Dockers $^{\circledast}$, symbole du business casual clothing

Levi's $^{(\circledast)}$ et Dockers ${ }^{(*)}$ diversification des circuits de distribution en Europe

Levi Strauss Signature ${ }^{(\circledast)}$ : une nouvelle marque pour la grande distribution

Levi Strauss Signature se différencie de Levi's

La marque est lancée d'abord en Amérique du Nord, en Australie et au Japon
La marque traditionnelle Levi's ${ }^{\circledR}$ est la principale marque de l'entreprise. Créée en 1873, elle est considérée comme l'une des plus grandes marques à l'échelle mondiale : en 2006, sa valeur est estimée à 2,69 milliards \$ (Interbrand, 2006). Levi's $^{\circledR}$ représente la première marque de jeans aux Etats-Unis : presque $70 \%$ des hommes américains, âgés de 15 à 49 ans, portent des jeans Levi's ${ }^{\circledR}$. Les produits commercialisés sous la marque Levi's ${ }^{\circledR}$ comprennent des vêtements pour hommes, femmes et enfants. Ils s'adressent à différents segments de clientèle et sont vendus via plusieurs circuits de distribution. La marque est commercialisée dans 110 pays. En 2005, le chiffre d'affaires réalisé est de 2,9 milliards $\$$, ce qui correspond à environ $71 \%$ du CA total du groupe.

La marque Dockers ${ }^{\circledR}$ concerne des vêtements de loisirs, principalement des pantalons et des T-shirts, vendus dans plus de 50 pays. La marque a joué un rôle important dans la diffusion des pantalons khaki et de la mode casual sur le lieu de travail aux Etats-Unis, en créant le «business casual clothing». Dockers ${ }^{\circledR}$ est la première marque de pantalons pour hommes aux Etats-Unis : presque $75 \%$ des hommes américains qui portent des pantalons casual achètent des Dockers ${ }^{\circledR}$. En 2005, le chiffre d'affaires réalisé par les produits de la marque s'élève à 784 millions $\$$, soit $19 \%$ du CA total du groupe.

Aux Etats-Unis, les marques Levi's ${ }^{\circledR}$ et Dockers ${ }^{\circledR}$ sont principalement distribuées à travers les magasins de chaîne et les grands magasins. A l'étranger, elles sont essentiellement vendues par les grands magasins et les détaillants indépendants. Les produits Levi's ${ }^{\circledR}$ et Dockers ${ }^{\circledR}$ sont également distribués par un nombre restreint de magasins possédés par l'entreprise et localisés aux Etats-Unis, en Europe et en Asie, et par environ 950 magasins franchisés localisés en dehors des Etats-Unis. En raison du caractère fragmenté du système de distribution européen, l'entreprise travaille avec différents types de distributeurs : les marques Levi's ${ }^{\circledR}$ et Dockers ${ }^{\circledR}$ sont commercialisées dans les grands magasins (par exemple les Galeries Lafayette en France, Kaufhof et Karstadt en Allemagne, El Corte Inglès en Espagne), les magasins Levi's ${ }^{\circledR}$ et Dockers ${ }^{\circledR}$, la vente par correspondance, et un nombre important de détaillants indépendants détenant un ou plusieurs magasins de jeans ou de vêtements.

Le lancement mondial de la nouvelle marque Levi Strauss Signature ${ }^{\circledR}$ vise à conquérir de nouveaux clients qui cherchent à acheter des vêtements de marque, mais qui sont sensibles au rapport qualité/prix. Les produits de la nouvelle marque couvrent des pantalons, des T-shirts et des vestes pour hommes, femmes et enfants. L'objectif prioritaire consiste à vendre des jeans en grande distribution : "sell jeans where people shop ». La marque s'adresse principalement aux consommateurs qui font leurs courses dans les magasins de la grande distribution. Aux Etats-Unis, ces derniers représentent le canal de distribution le plus important pour les jeans : près de $30 \%$ des ventes y sont réalisées.

Comparés aux produits de la marque Levi's ${ }^{\circledR}$, les jeans Levi Strauss Signature ${ }^{\circledR}$ remplissent les mêmes exigences concernant la qualité des produits, mais ils présentent moins d'effets spéciaux tels que 'l'effet d'usure' qui peut représenter de quatre à dix euros du prix de vente des jeans commercialisés sous la marque Levi's ${ }^{\circledR}$. Par ailleurs, ils ne portent pas les signes emblématiques de Levi's $^{\circledR}$, comme le fameux «Red $T a b$ », l'étiquette en cuir avec les deux chevaux et la couture arquée sur les poches arrières. A travers ces différences, le groupe cherche à éviter le risque de cannibalisation avec la marque Levi's ${ }^{\circledR}$.

Levi Strauss Signature ${ }^{\circledR}$ est lancée en juillet 2003 : aux Etats-Unis, les produits sont d'abord commercialisés par 3000 magasins Wal-Mart avant d'être vendus dans les magasins Target et Kmart. La marque est ensuite introduite au Canada (où elle est commercialisée par les magasins Wal-Mart), en Australie (où un accord a été signé avec les magasins Lowes et Big W) et au Japon (où les produits sont vendus chez Justco et Saty). En 2003, le chiffre d'affaires réalisé par la marque Levi Strauss Signature ${ }^{\circledR}$ s'élève à 0,2 milliard $\$$, ce qui représente environ $6 \%$ du chiffre d'affaires total du groupe. 
Afin de maintenir son leadership sur le marché des vêtements de marque, le groupe a adopté quatre principes : (1) créer des produits innovants et adaptés aux consommateurs, en relançant des produits existants et en introduisant de nouveaux produits qui peuvent être rapidement commercialisés à travers différents canaux de distribution ( innovate and lead from the core »); (2) améliorer de façon constante les flux logistiques et les flux de commercialisation afin de répondre plus efficacement et plus rapidement aux évolutions du marché, en poursuivant l'objectif de réduction des coûts ("achieve operational excellence ") ; (3) mener des opérations communes, améliorer les marges des distributeurs et rendre les produits plus faciles d'accès pour les consommateurs ( « revitalise retailer relationships and improve the company's presence at retail »); (4) rendre les produits accessibles grâce à des canaux de distribution multiples et grâce à des prix qui correspondent aux attentes des consommateurs ( "sell where they shop»).

\section{La délicate approche du marché européen}

La filiale européenne du groupe Levi Strauss est située à Bruxelles. Son Président, Paul Mason, de nationalité britannique, a acquis une solide expérience dans la grande distribution. En 2005, la filiale européenne a réalisé un chiffre d'affaires de 981 millions \$. Le groupe a récemment réorganisé ses activités en Europe : l'objectif est de décentraliser et de redonner plus de responsabilités à chaque pays plutôt que de considérer la région sur une base pan-européenne, comme ce fut le cas au début. II est vrai que la principale difficulté, pour une entreprise originaire d'un pays tiers, est de prendre la mesure de l'hétérogénéité d'un marché européen où chaque pays présente des caractéristiques spécifiques (législation, comportements diversifiés des consommateurs liés aux différences culturelles, etc.). Indépendamment de ce changement d'approche, l'entreprise cherche à repositionner en Europe la marque Levi's ${ }^{\circledR}$ sur le traditionnel segment premium - contrairement au marché américain où les jeans Levi's ${ }^{\circledR}$ sont positionnés comme des 'jeans ordinaires', ainsi vendus à des prix inférieurs à ceux pratiqués en Europe. Une campagne de communication mettant en relief le style des jeans 501 vise à attirer l'attention des jeunes consommateurs dans le but de stimuler les ventes en Europe. La filiale bruxelloise a également entamé un vaste programme de réduction des coûts afin d'améliorer les marges réalisées. Ce programme concerne les activités de production, mais aussi la commercialisation des produits.

En 2004, la marque Levi Strauss Signature ${ }^{\circledR}$ est introduite sur le marché européen. La filiale européenne est chargée d'identifier les marchés qui représentent le potentiel le plus important et d'élaborer une politique marketing cohérente pour les pays sélectionnés. Si la politique marketing proposée doit prendre en considération les choix effectués sur les autres marchés géographiques, la filiale dispose d'un certain degré d'autonomie afin de répondre aux spécificités du contexte européen. Elle décide de se focaliser, dans un premier temps, sur les grands marchés européens du textile-habillement. Pour sélectionner ceux qui représentent le potentiel le plus important, elle analyse les caractéristiques du marché des Big Five, constitué par l'Allemagne, la France, le Royaume-Uni, l'Italie et l'Espagne.

Les caractéristiques du marché des «Big Five»

\begin{tabular}{|c|c|c|c|c|c|c|}
\hline & Allemagne & France & Royaume-Uni & Italie & Espagne & Total \\
\hline Nombre d'habitants (en millions) & 83 & 60 & 59 & 57 & 41 & 300 \\
\hline P.I.B. (en milliards de \$) & 1846 & 1310 & 1424 & 1089 & 582 & 6251 \\
\hline P.I.B. par habitant (en \$) & 22418 & 21990 & 24186 & 18928 & 14234 & - \\
\hline Nombre de jeans vendus (en millions) & 84,4 & 52,3 & 53,9 & 29,7 & 27,2 & 247,5 \\
\hline $\begin{array}{l}\text { Nombre de jeans vendus en grande } \\
\text { distribution (en millions) }\end{array}$ & 6,5 & 14,9 & 13,4 & 2,5 & 2,7 & 40 \\
\hline
\end{tabular}

Source : Levi Strauss \& Co (2006), Internal Documents. 
Le choix porte sur la France, le Royaume-Uni..

... et l'Allemagne, malgré le faible poids de la grande distribution

Une stratégie marketing unique pour ces trois pays

Les produits commercialisés sont identiques sur les trois marchés

En revanche, les prix sont différenciés
Après un examen approfondi du marché des Big Five, la filiale européenne décide d'introduire la nouvelle marque dans les trois plus grands pays européens : l'Allemagne, la France et le Royaume-Uni. Ces trois marchés comptent plus de 200 millions d'habitants et se caractérisent par un pouvoir d'achat élevé. Il s'agit également des plus importants marchés pour les jeans : près de 190 millions de jeans y sont vendus chaque année. La France et le Royaume-Uni représentent le potentiel le plus fort en matière de vente de jeans en grande distribution: respectivement 14,9 millions de jeans (soit $28 \%$ des ventes de jeans) et 13,4 millions (soit $25 \%$ des ventes de jeans) y sont vendus dans les circuits de la grande distribution.

En Allemagne, le potentiel de la grande distribution pour la vente de jeans paraît plus faible (6,5 millions de jeans vendus, soit $8 \%$ du marché), bien qu'il soit supérieur à ce qu'il est en Italie et en Espagne. En effet, les Allemands préfèrent acheter les vêtements dans les grands magasins et les magasins spécialisés. Par ailleurs, la grande distribution y est moins développée qu'en France et au Royaume-Uni, compte tenu notamment du poids du hard-discount (Guiot, 2004). Le potentiel du marché allemand paraît néanmoins important, car le nombre de jeans vendus chaque année est supérieur au nombre d'habitants.

La filiale européenne met au point une stratégie marketing pour les trois pays sélectionnés. La segmentation du marché s'effectue en fonction de deux critères. Le premier est l'âge des personnes (qui influence le style vestimentaire privilégié), le second, le lieu d'achat : bien que les magasins spécialisés constituent le principal canal de distribution pour la vente de jeans, les consommateurs ont des pratiques différenciées concernant l'endroit où ils achètent les jeans. Français et Britanniques montrent un intérêt grandissant pour la grande distribution. Le cœur de cible est constitué par les 25 à 35 ans qui sont soucieux d'un bon rapport qualité/prix, mais qui cherchent à acheter de vraies marques; la marque souhaite conquérir les clients de la grande distribution susceptibles d'y acheter des jeans à condition qu'ils soient de qualité. Pour positionner la nouvelle marque, l'entreprise met en avant trois caractéristiques principales du produit : la qualité, le style et l'héritage pour des consommateurs sensibles au rapport qualité/prix. L'héritage fait référence au mythe du Far West et «au rêve américain » qui séduisent beaucoup de consommateurs européens, et plus particulièrement les Allemands qui ont une image très positive de l'American dream depuis l'accélération de l'industrialisation à la fin du XIX siècle ; la libération par les forces américaines en 1945, puis la large diffusion, après 1968, des valeurs de liberté et d'ouverture au monde l'ont accentuée.

La filiale européenne choisit de standardiser la politique de produit adoptée sur le marché européen. Les attributs des produits Levi Strauss Signature ${ }^{\circledR}$ sont identiques à travers l'Europe, ce qui permet la réalisation d'économies d'échelle et le développement d'une image de marque uniforme (Ghauri et Cateora, 2005 ; Mayrhofer, 2004). Le nom de marque reste également le même, mais il est prononcé de manière différente en Allemagne, en France et au RoyaumeUni. En Allemagne, le nom est prononcé en anglais, ce qui permet d'asseoir l'origine américaine de la marque, alors qu'en France, la prononciation est 'francisée'. II paraît intéressant de noter que le nom de marque Levi Strauss Signature $^{\circledR}$ bénéficie d'une certaine notoriété que l'entreprise qualifie de « notoriété fantôme » (ghost awareness). En effet, comme la nouvelle marque comporte le nom « Levi Strauss », elle bénéficie automatiquement d'un certain degré de notoriété : les consommateurs pensent connaître la nouvelle marque, parce qu'ils connaissent la marque Levi's ${ }^{\circledR}$.

L'entreprise choisit d'adapter les prix des produits Levi Strauss Signature ${ }^{\circledR}$ à chaque pays, notamment en raison de la sensibilité des consommateurs par rapport au prix et en raison des marges appliquées par les distributeurs. Ainsi, les prix en France sont supérieurs à ceux pratiqués en Allemagne où les consommateurs sont particulièrement sensibles au prix (Guiot, 2004), d'autant 
qu'ils possèdent déjà au moins un jean chacun en moyenne. Les Français accordent souvent plus d'importance à la marque qu'au prix, notamment dans le domaine du textile-habillement. Mais les prix fixés en France restent inférieurs à ceux du marché britannique où les marges commerciales de la grande distribution sont supérieures de $10 \%$ par rapport à la France. Les prix adoptés pour les jeans Levi Strauss Signature ${ }^{\circledR}$ sur le marché européen varient ainsi entre 25 et $40 €$, selon les modèles proposés et selon les promotions offertes par les distributeurs. La fourchette de prix adoptée permet une différenciation claire entre les jeans Levi's ${ }^{\circledR}$ (vendus à plus de $50 €$ sur le marché européen) et Levi Strauss Signature ${ }^{\circledR}$. La différence de prix est d'environ $30 \%$, ce qui correspond à la différence habituelle de prix entre une grande marque et une marque de distributeur, avec l'avantage stratégique que Levi Strauss Signature ${ }^{\circledR}$ appartient à la catégorie des grandes marques.

En raison du caractère hétérogène des systèmes de distribution européens, l'entreprise choisit de travailler avec plusieurs distributeurs. Elle décide de collaborer avec un nombre limité d'entre eux dans chaque pays afin d'établir des relations étroites avec chaque enseigne. Comme le partenariat engagé avec Wal-Mart aux Etats-Unis a permis d'obtenir des résultats satisfaisants, Levi Strauss étend l'accord signé avec Wal-Mart aux marchés allemand et britannique. La filiale européenne noue également des partenariats avec d'autres distributeurs afin d'obtenir une couverture géographique satisfaisante pour la distribution des produits.

Les choix effectués montrent que le groupe privilégie de travailler avec quelques grandes enseignes présentes sur les marchés internationaux afin de limiter le nombre de partenaires et afin d'assurer une certaine cohérence au niveau de la politique marketing menée. En effet, le distributeur américain WalMart, grâce à une politique d'acquisitions, s'est hissé au rang de premier distributeur à l'échelle mondiale (Lehmann-Ortega et Schoettl, 2004) : il est présent au Royaume-Uni (à travers 265 magasins Asda) et en Allemagne (à travers 89 magasins Wal-Mart). Le français Carrefour constitue le deuxième distributeur à l'échelle mondiale, et possède 210 hypermarchés en France et 314 en Europe.

En France, la marque Levi Strauss Signature ${ }^{\circledR}$ est donc, dans un premier temps, commercialisée dans les hypermarchés Carrefour. Au Royaume-Uni, elle est référencée dans les magasins Asda, Wal-Mart et Tesco (qui détient une part de marché de $30 \%$ ). En Allemagne, elle est vendue dans les magasins Wal-Mart, mais aussi dans les points de vente de l'enseigne Handelshof (un distributeur de masse qui offre un large choix de produits, essentiellement dans le domaine alimentaire et qui coopère avec Edeka), ceci en raison de la faible présence de Wal-Mart dans certaines régions. Le groupe Wal-Mart a récemment cédé ses magasins en Allemagne au groupe Metro, notamment en raison des difficultés rencontrées par l'enseigne américaine outre-Rhin : les prix affichés étant souvent supérieurs à ceux pratiqués dans les magasins du harddiscount comme Aldi et Lidl, l'enseigne n'avait pas de légitimité sur le créneau du bas prix dans un pays où le poids du hard-discount (plus de $36 \%$ de part de marché) est trois fois plus important qu'en France. A cela s'ajoutaient des erreurs de management manifestes liées à la mécompréhension des différences culturelles.

L'objectif principal de la communication consiste à inciter les clients de la grande distribution à acheter des jeans dans ce canal de distribution et à choisir la marque Levi Strauss Signature ${ }^{\circledR}$. En effet, selon une enquête réalisée par l'entreprise, seulement $14 \%$ des clients de la grande distribution en Europe ont I'habitude d'y acheter des vêtements (y compris des jeans) ; $54 \%$ des personnes interrogées envisagent d'acheter des vêtements ( $y$ compris des jeans) en grande distribution, et $32 \%$ déclarent ne pas vouloir y acheter de vêtements. La communication du groupe vise dès lors à convaincre les deux premiers groupes de consommateurs à acheter des jeans de la marque Levi Strauss
L'entreprise décide de collaborer avec plusieurs distributeurs ...

... qui sont présents sur les marchés internationaux

... et qui permettent une couverture géographique satisfaisante

La communication s'adresse aux clients de la grande distribution 
Elle est réalisée en collaboration avec la grande distribution

Le succès de la politique marketing varie suivant les marchés

Spécificités de la structure de la distribution en Allemagne
Signature ${ }^{\circledast}$. Le marché potentiel paraît d'autant plus important que la grande distribution offre un choix limité au niveau des vêtements, notamment pour les jeans, et commercialise essentiellement ses propres marques.

La filiale européenne choisit de centrer la communication sur les valeurs véhiculées par la marque : la qualité, le style et l'héritage. Elle utilise les supports nationaux de la presse spécialisée destinée aux distributeurs. Par ailleurs, elle négocie des espaces publicitaires dans les brochures des différents distributeurs afin de communiquer envers les consommateurs. Dans cette perspective, il paraît nécessaire de préciser que les supports de communication continuent à varier suivant les pays et peuvent avoir des audiences très variables (Tellefsen et Takada, 1999). Par exemple, en Allemagne où l'offre en journaux et magazines est particulièrement riche et diversifiée, le poids de la publicité dans la presse écrite est plus important qu'en France et au Royaume-Uni.

La politique marketing mise en place par la filiale européenne du groupe Levi Strauss rencontre un succès important auprès des distributeurs et auprès des consommateurs : en 2004, année du lancement, les ventes dépassent les prévisions de $18 \%$ (les résultats enregistrés par Carrefour France sont même supérieurs de $35 \%$ aux objectifs fixés), et la nouvelle marque affiche des résultats bénéficiaires. Les distributeurs se montrent satisfaits de la relation établie avec le groupe Levi Strauss : le référencement de la nouvelle marque leur a notamment permis d'accroître les ventes dans le domaine du textile-habillement. En effet, le fait de commercialiser des marques incite les consommateurs à acheter des jeans et d'autres vêtements dans les grandes surfaces. Pour les années 2005 et 2006, si l'entreprise ne communique pas de chiffres précis, elle remarque néanmoins que les ventes réalisées par l'entreprise en France et au Royaume-Uni sont supérieures à celles enregistrées en Allemagne. Le succès mitigé de la marque Levi Strauss Signature ${ }^{\circledR}$ sur le marché allemand peut être attribué à deux facteurs principaux : la structure du système de distribution et le comportement d'achat des consommateurs.

\section{Le marché allemand du textile-habillement : spécificités et tendances}

Sur le marché allemand, le rôle joué par la grande distribution dans la vente de jeans et d'autres vêtements reste limité. Contrairement à la France et au Royaume-Uni où la distribution est marquée par la prédominance des hypermarchés, véritables 'généralistes' qui disposent tous d'un rayon textile (avec une offre variée, des cabines d'essayage, etc.), la distribution en Allemagne présente une structure très différenciée. Les hypermarchés au sens français (caractérisés en outre par leur situation excentrée) sont un phénomène qui n'a commencé à apparaître qu'après l'unité allemande, et restent marginaux. Par ailleurs, deux types de circuits de distribution se distinguent: d'une part, le "grand magasin " (Kaufhaus). II offre certes une vaste gamme de produits, mais reste spécialisé dans le textile-habillement et l'équipement de la maison, l'alimentaire ne constituant qu'une diversification a posteriori, développée par certaines enseignes (comme Karstadt ou, leader incontesté, le KaDeWe de Berlin). C'est dans ces grands magasins que les Allemands achètent la plupart de leurs vêtements ; ils sont situés en plein centre ville. D'autre part, il existe le Supermarkt, format du supermarché (comme Edeka et Handelshof) à large prédominance alimentaire. Ces magasins proposent, tendance relativement récente à la diversification dans le non food, un choix réduit d'articles textiles et doivent affronter la concurrence du hard-discount qui multiplie les offres promotionnelles au niveau des vêtements. Sur le marché allemand, Aldi et Lidl figurent respectivement au septième et huitième rang des distributeurs du textilehabillement. Parallèlement, on peut observer l'importance grandissante des magasins discount spécialisés dans le textile-habillement comme Takko et Kik qui proposent des vêtements à bas prix (KPMG, 2006). 
C'est sur le segment de distribution Supermarkt que s'est positionné Wal-Mart. Les difficultés rencontrées par l'enseigne, le principal partenaire de l'entreprise en Allemagne, ont sans doute affecté les ventes de la marque Levi Strauss Signature ${ }^{\circledR}$. L'acquisition récente des magasins Wal-Mart par le groupe Metro (qui constitue le troisième distributeur du textile-habillement outre-Rhin derrière KarstadtQuelle et le groupe Otto) pourrait accroître les ventes de la marque. D'autant que, selon une récente enquête de l'institut GfK, plus de $70 \%$ des Allemands ont acheté au moins une fois des T-shirts, des pantalons ou autres vêtements dans un de ces supermarchés, dont Edeka (plus de 3000 points de vente en Allemagne). Près d'un tiers y effectue régulièrement des achats vestimentaires. Le concept du « supermarché » présente, semble-t-il, un fort potentiel pour le jean et autres vêtements de bonne qualité : plus de la moitié des clients les fréquentent régulièrement pour 'jeter un œil' sur l'offre textile et, le cas échéant, effectuer un achat spontané.

Le succès limité enregistré jusqu'ici par la marque Levi Strauss Signature ${ }^{\circledR}$ sur le marché allemand s'explique aussi par le comportement d'achat des consommateurs : d'une part, ceux-ci cherchent à acheter des vêtements à des prix discount, d'autre part, ils sont prêts à dépenser des sommes importantes pour des produits de marque. De ce fait, le marché allemand se caractérise par une forte polarisation entre la mode "fashion" et "classique " (vêtements de marque commercialisés essentiellement dans les grands magasins et les magasins spécialisés) et la mode à bas prix (vendue dans les magasins discount), une tendance qui va probablement s'accentuer dans les années à venir. Parallèlement, on peut observer que les Allemands sont devenus moins fidèles à une enseigne: pour chaque vêtement acheté, ils fréquentent, en moyenne, six points de vente (KPMG, 2006).

Mais ces tendances globales demandent à être nuancées. Certes, les Allemands, dont le pouvoir d'achat stagne depuis plusieurs années, sont en quête de bas prix; et, comme le rappelle l'institut GfK, soldes et promotions représentent le quart des vêtements achetés outre-Rhin. Mais les clients, saturés par les stratégies promotionnelles ininterrompues des discounters ou non, adaptent leurs comportements. Ils ne sont plus en quête de la promotion en tant que telle; ils cherchent aussi la qualité. La relation qualité/prix gagne d'autant plus en importance à leurs yeux qu'ils sont nombreux à posséder des vêtements 'basiques' acquis à prix modiques, dont des jeans. Cela vaut particulièrement pour les plus jeunes, cœur de cible du groupe Levi Strauss. S'y ajoute la montée en puissance du groupe des "smart shoppers": ces consommateurs aux revenus moyens, voire supérieurs, qui ont développé une nette prédilection pour les bas prix pratiqués dans l'habillement par Aldi et Lidl, mais aussi Edeka ou Tengelmann, car ils ont confiance dans la qualité des produits vendus, interprétant ces prix avantageux comme le fruit "d'une politique d'achat intelligente » de la part des distributeurs (GfK Textil News, été 2006).

Meme SI LA REUSSITE DE LA NOUVELle maRQue dépend des relations engagées avec la grande distribution, les perspectives futures paraissent intéressantes. Ainsi, le groupe envisage d'élargir son réseau de distributeurs sur les marchés existants et de développer la marque sur de nouveaux marchés. La filiale européenne a récemment noué des accords avec les enseignes Auchan et Géant en France, Migros en Suisse, Carrefour en Belgique, Bilka au Danemark, KCitymarket en Finlande et Auchan en Espagne. Mais le groupe prévoit aussi de développer la marque dans d'autres régions géographiques, notamment en Amérique Latine et en Asie. L'analyse du marché européen montre combien il est important de prendre en considération les spécificités de chaque pays, notamment au niveau de la structure du système de distribution et du comportement d'achat des consommateurs.

Après un départ difficile en Allemagne, le lancement de Levi Strauss Signature ${ }^{\circledR}$ pourrait déboucher sur un succès si la stratégie marketing suit l'évolution des
Montée en puissance du supermarché pour l'achat vestimentaire

Les Allemands achètent des vêtements à prix discount ou des vêtements de marque

Mais ils cherchent aussi de plus en plus la qualité à prix modique 
tendances chez les consommateurs de même que celles de la distribution. $\mathrm{Si}$ on excepte Wal-Mart, à l'avenir pour l'instant incertain, le groupe américain semble bien positionné outre-Rhin en misant sur Handelshof et son partenaire Edeka, à l'offre de prix légèrement supérieure au hard discount. Car visiblement, les activités non food des géants du discount Aldi et Lidl ont dépassé leur zénith (GfK, communiqué du 16-03-07). Depuis 1998, leurs parts de marché dans ce segment ne cessaient de croître, cumulant à $5 \%$ en 2005. En 2006, elles sont tombées à $4,6 \%$, toutes catégories de produits confondues. Cette tendance est durable, selon les prévisions de l'institut GfK. Aldi et Lidl, qui avaient lancé une politique de promotion bi-hebdomadaire, suivis par tous les autres discounters, paient aujourd'hui le fait qu'il s'agit d'opérations uniques et que les produits, pourtant dotés d'une bonne image en termes de qualité, ne sont pas suivis. Par ailleurs, la pléthore des 'promos' a abouti à une saturation du marché et à la 'désensibilisation' des consommateurs. S'ils sont toujours en quête de la 'bonne affaire', les Allemands tendent nettement à préférer désormais la qualité. Si de surcroît le groupe américain parvient à diffuser astucieusement les valeurs liées à sa nouvelle marque, il entrerait également en concordance avec cette tendance lourde de la société allemande au "re-grounding »: la réappropriation des valeurs traditionnelles désormais chargées de connotations nouvelles (voir REA 80/07). La valeur " héritage », en tout cas, pourrait bénéficier de cette tendance au 'retour aux sources' qui caractérise les consommateurs allemands, y compris le cœur de cible de la marque.

\section{Indications bibliographiques}

Ghauri P. et Cateora PH. (éds.), International Marketing. European Edition, $2^{\mathrm{e}}$ éd., Londres, McGraw Hill, 2005

GuIot, C., "Le phénomène discount en Allemagne ", Regards sur l'économie allemande, $n^{\circ} 67,2004$

INTERBRAND (2006), Best Global Brands 2006. A Ranking by Brand Value

KPMG (2006), Trends im Handel 2010

LEHMANN-ORTEGA L. et SCHOETTL J.-M., "Distribution et modèles d'internationalisation », L'Expansion Management Review, 2004

LeVI Strauss \& Co, Annual Financial Report, 2005

LeVI STRAuSs \& Co, Internal Documents, 2006

MAYRHOFER U., Marketing International, Paris, 2004

Tellefsen T. et TAKADA H., " The Relationship between Mass Media Availability and the Multicountry Diffusion of Consumer Products ", Journal of International Marketing, vol. 7, n¹, 1999 www.gfk.de (Consumer Tracking). 